\title{
Fat and lean tissue accretion in relation to reward motivation in
}

\section{children}

De Decker Annelies ${ }^{\mathrm{a}}$, De Clercq Bart ${ }^{\mathrm{a}}$, Verbeken Sandra ${ }^{\mathrm{b}}$, Wells Jonathan C. K. ${ }^{\mathrm{c}}$, Braet Caroline $^{\mathrm{b}}$, Michels Nathalie ${ }^{\mathrm{a}}$, De Henauw Stefaan ${ }^{\mathrm{a}}$, Sioen Isabelle $^{\mathrm{a}}$

\section{Author affiliations:}

${ }^{\text {a } D e p a r t m e n t ~ o f ~ P u b l i c ~ H e a l t h, ~ F a c u l t y ~ o f ~ M e d i c i n e ~ a n d ~ H e a l t h ~ S c i e n c e s, ~ G h e n t ~ U n i v e r s i t y, ~ D e ~ P i n t e l a a n ~} 185$ UZ 4K3, 9000 Gent, Belgium. Annelies.DeDecker@UGent.Be, Isabelle.Sioen@UGent.Be,

Nathalie.Michels@UGent.Be, Stefaan.DeHenauw@UGent.Be.

${ }^{\mathrm{b}}$ Department of Developmental, Personality and Social Psychology, Faculty of Psychology and Educational Sciences, Ghent University, Henri Dunantlaan 2, 9000 Gent, Belgium. Sandra.Verbeken@UGent.Be,

Caroline.Braet@UGent.Be.

${ }^{\mathrm{c}}$ Childhood Nutrition Research Centre, Institute of Child Health, University College of London, 30 Guilford Street, WC1N 1EH, London, United Kingdom. Jonathan.Wells@UCL.ac.UK.

\section{Corresponding author:}

De Decker Annelies

Annelies.DeDecker@UGent.Be 


\section{Abstract}

'Reward sensitivity' explains inter-individual differences in the motivation to obtain rewards when reward cues are perceived. This psychobiological trait has been linked to higher consumption of palatable food when exposed to palatable food cues. The current study aims to examine if reward sensitivity explains differences in patterns of fat and lean tissue accretion over time in children. A longitudinal observational study with measurement waves in 2011 (baseline), 2012, 2013, and 2015 was conducted. The sample was a population-based Flemish cohort of children ( $n=446,50 \%$ boys and 5.5 to 12 years at baseline; $38.8 \%$ of the baseline sample also participated in 2015). Baseline reward sensitivity of the children was assessed by parent ratings on the Drive subscale of the Behavioral Inhibition System/Behavioral Approach System scales. Age- and sex-independent Fat and Lean Mass Index z-scores (zFMI and zLMI respectively) were computed for each study wave based on air-displacement plethysmography. In girls, but not boys, reward sensitivity was positively associated with the baseline zFMI and zLMI (95\% confidence intervals of unstandardized estimates: 0.01 to 0.11 and 0.01 to 0.10 respectively, $\mathrm{P}$ values 0.01 and 0.02 respectively). Further, reward sensitivity explained $14.8 \%$ and $11.6 \%$ of the change in girls' zFMI and zLMI respectively over four years: the zFMI and zLMI increased and decreased respectively in high reward sensitive girls (95\% confidence intervals of unstandardized estimates: 0.01 to 0.11 and -0.12 to -0.01 respectively, $\mathrm{P}$ values 0.01 and 0.02 respectively). Hence, girls high in reward sensitivity had significantly higher adiposity gain over four years parallel with lower increase in lean mass than was expected on the basis of their age and height. These results may help to identify appropriate targets for interventions for obesity prevention.

\section{Keywords}

Reward; child; fat mass; lean mass; overweight; longitudinal.

\section{Introduction $^{1}$}

The psychobiological trait 'Reward sensitivity' (RS) explains inter-individual differences in motivation to approach rewards in response to reward-predicting-cues (Carver \& White, 1994). Rewards can be presents,

\footnotetext{
${ }^{1}$ Abbreviations: RS, reward sensitivity; BMI, Body Mass Index; FMI, Fat Mass Index; LMI, Lean Mass Index; zBMI, standard deviation score of BMI based on Flemish reference curves; zFMI, standard deviation score of FMI based on British reference curves; zLMI, standard deviation score of LMI based on British reference curves; BIS/BAS, Behavioral Inhibition System/Behavioral Approach System; AIC, Akiake's Information Criterion.
} 
money, social factors (e.g. appraisal), palatable foods (such as sweet and fat rich foods) etc. Individuals learn that the possibility to obtain rewards is associated with certain cues (objects, pictures, smells etc.). These cues can trigger a motivated state in individuals that aims to generate behavior to approach and obtain the associated reward. Applied to palatable food, cues associated with the consumption of palatable food can evoke the tendency to approach and consume these foods, even in the absence of homeostatic hunger (Johnson, 2013). Individuals high in RS have a stronger tendency to approach and obtain rewards when perceiving rewardpredicting-cues (Carver \& White, 1994). Therefore, it has been proposed that RS contributes to the variability in adiposity gain in contexts in which food cues are highly present in the daily living environment (e.g. billboards, commercials, vending machines) (Davis, Strachan, \& Berkson, 2004).

Considering the current high overweight and obesity prevalence in childhood (Lobstein, Baur, \& Uauy, 2004) and its tracking into adulthood (Singh, Mulder, Twisk, van, \& Chinapaw, 2008), the development of effective childhood obesity prevention strategies is of great importance. To identify appropriate targets for obesity prevention interventions, confirming and quantifying the association between RS and adiposity gain in children is critical. Different approaches to measure inter-individual differences in cue-triggered reward motivation exist, e.g. neuro-imaging techniques, laboratory-based behavioral tasks, and questionnaires that measure RS to all kinds of reward, i.e. 'general RS', as well as specifically to palatable food reward (Carnell, Benson, Pryor, \& Driggin, 2013). To use the information on this association for preventive purposes, the RS measure needs to be easily and practically applicable, and inexpensive; therefore, it is advisable to use questionnaires.

Using a general RS questionnaire, cross-sectional studies have reported higher consumption of palatable food (De Cock et al., 2016; De Decker et al., 2016; Paquet et al., 2010), food cravings (Franken \& Muris, 2005), over-eating (Davis et al., 2007), and overweight (Davis \& Fox, 2008; De Decker et al., 2016; Dietrich, Federbusch, Grellmann, Villringer, \& Horstmann, 2014; Verbeken, Braet, Lammertyn, Goossens, \& Moens, 2012) with higher RS. Some of these studies differentiated by sex and reported that this relation was primarily present in females (De Cock et al., 2016; Dietrich et al., 2014). To confirm that higher scores on a general RS questionnaire indeed increase the risk of excess adiposity gain, longitudinal studies are needed but these are as far as we know absent. Therefore, the current study aimed to examine if inter-individual differences in general RS explain differences in fat and lean tissue accretion in children, and if the relation between RS and fat and lean tissue accretion differs in boys versus girls. The consideration of fat and lean tissue accretion instead of weight gain is an important advantage of this study, since lean tissue accretion can importantly contribute to weight gain 
(Wells, 2000). A positive association between RS and fat as well as between RS and lean tissue accretion over time was hypothesized, and it was hypothesized that this relation was stronger in girls.

\section{Methods}

\section{Participants and procedure}

Participants were 446 Dutch-speaking Belgian children (50\% boys) aged 5.5 to 12 years at baseline (i.e. 2011). Baseline data on parental reports of children's RS as well as measurements of children's body composition in 2011, 2012, 2013, and 2015 were used (figure 1). For all these study waves, children (in most cases accompanied by at least one parent) attended the survey center at a prefixed appointment, on which body composition measurements of the child were conducted and parental questionnaires were filled in. If no parent accompanied the child, one of the parents completed the questionnaires at home.

The children were recruited by random cluster design (all children of twelve primary schools of Aalter were contacted) for the longitudinal “Children's Body Composition and Stress" (ChiBS) study (Michels et al., 2012), with study surveys in 2010, 2011, and 2012. Data from 2010 were not used since RS was assessed for the first time in 2011. All children measured in the ChiBS study were invited via mail, email and, if no response, telephone calls to participate in the follow-up study "Rewarding-FOod ChoicES" (Forces), which is part of the REWARD-project (www.rewardstudy.be), with study surveys in 2013 and 2015. All participants for which baseline RS data were present were included in the current study (i.e. 446 of the 455 children that participated in the ChiBS survey of 2011).

The ChiBS and Forces studies were conducted according to the guidelines laid down in the Declaration of Helsinki and approved by the Ethics Committee of Ghent University Hospital. Written informed consent was obtained from all parents, and all children gave verbal assent.

\section{Measures}

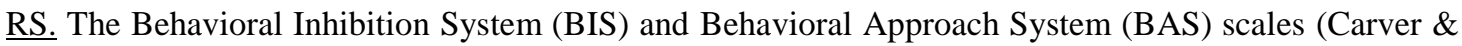
White, 1994) measure a person's behavioral and affective responses to (predicted) punishments and rewards respectively. The BAS scale consists of three subscales, i.e. Fun Seeking (measures the inclination to seek out 
new rewarding situations), Reward Responsiveness (measures positive affect and excitability when obtaining reward), and Drive (measures the strength of pursuit to obtain reward in response to reward-predicting cues; e.g. our child does everything to get the things that he/she wants). Hence, the Drive subscale is most strongly linked to the RS concept as described in the introduction section. Additionally, of the three BAS subscales, Drive has the highest internal consistency (De Cock et al., 2016; Vervoort et al., 2015) and correlates most strongly with neural responses to food reward cues in multiple loci of the brain reward circuitry $(r \sim 0.8)$ (Beaver et al., 2006). Therefore, the term RS in the text below refers to the sum score of the four items of the Drive subscale, which were scored on a 4-point Likert scale $(1=$ not true; $2=$ somewhat true; $3=$ true, $4=$ very true; total range on Drive subscale: 4-16). Because the youngest children of the cohort were too young to answer the questionnaire themselves, parents answered a Dutch parent version of the BIS/BAS scale (Vervoort et al., 2015). The Cronbach alpha of RS in the current study (0.85) was comparable with the alpha reported by Vervoort et al. (i.e. 0.85 ) in children and adolescents aged 2-18 years (Vervoort et al., 2015).

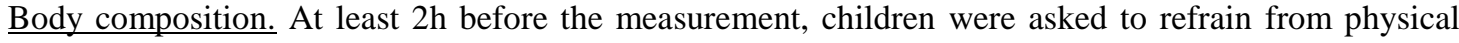
activity and food. Height was measured to the nearest $0.1 \mathrm{~cm}$. Body weight was measured with the BOD POD ${ }^{\circledR}$ balance, and body volume with the BOD POD® air-displacement plethysmography device (Software version 4.2.4, Life Measurement, Inc., Concord, California, USA), both using standardized procedures (McCrory, Gomez, Bernauer, \& Mole, 1995). In accordance to the manufacturer's guidelines, the BOD POD® was calibrated daily and at each measurement, and children wore tight-fitting bathing suits with swimming caps during the measurement. Thoracic gas volume was predicted by the software with a validated child-specific equation (Fields, Hull, Cheline, Yao, \& Higgins, 2004). Using weight, body volume, and thoracic gas volume, body density was calculated and converted into fat and lean masses using child-specific conversion factors (Wells et al., 2010). Then, fat and lean mass (kilogram) were divided by height squared (meter ${ }^{2}$ ) to calculate fat mass index (FMI) and lean mass index (LMI), each in the same kilogram/meter ${ }^{2}$ units as body mass index (BMI). Since no Flemish FMI and LMI reference curves for children are available, British reference curves were used to compute age- and sex-independent standard deviation scores of FMI (zFMI) and LMI (zLMI) (Wells et al., 2012). For descriptive purposes, also BMI was calculated and z-scores were computed based on Flemish reference curves (Roelants, Hauspie, \& Hoppenbrouwers, 2009).

\section{Data analyses}


The two-sided level of significance was set at $\mathrm{p}<0.05$. Descriptive analyses were performed using PASW Statistical Program version 20.0 (SPSS, IBM, IL, USA). Unpaired t-tests were conducted to test sex differences on age, RS, zFMI, and zLMI. To test drop-out differences, participants versus non-participants were compared on sex with a chi-square test and on age, RS, zFMI and zLMI with unpaired t-tests.

Longitudinal multilevel analyses were conducted in Mplus version 7.20 to examine change in zFMI and zLMI across time (Muthén \& Muthén, 1998-2012). All models were calibrated using the default maximum likelihood estimation with robust standard errors. Multilevel modelling has significant advantages over traditional repeated measure techniques, since this does not require balanced data (i.e. number of available measurements do not have to be the same for all individuals) (Hox, 2010). A two-level model was used, with the repeated measures on the four time points at the lowest level (i.e. time level), and individual child parameters at the highest level (i.e. child level).

Preliminary multilevel analyses showed that sex significantly moderated the main effect of RS on zFMI. Furthermore, zLMI followed a different pattern in boys versus girls over time (Table 1), possibly due to slight differences in the accretion of lean mass between Flemish and British children. Therefore, the multilevel analyses were performed separately for boys and girls.

Four parallel series of incremental models were conducted, i.e. series for girls' zFMI change, series for girls' zLMI change, series for boys' zFMI change and series for boys' zLMI change. The incremental models, identical for each series, are explained in the following. Model 1 contained only the measurement occasion (time, split up in three time dummies) on the time level; this model provides the intraclass correlation (Hox, 2010). In model 2, the baseline centralized RS score (i.e. RS minus 9) was added on the child level. In model 3 , the coefficients of the time dummies were allowed to be random. This model allows to evaluate the significance of the variance of the zFMI or zLMI slopes between time points (i.e. differences in the slopes of zFMI and zLMI between children over the time spans baseline-2012, baseline-2013 or baseline-2015). In model 4, cross-level interaction terms between RS and the time dummies were computed to assess whether RS explains differences in the slopes of zFMI and zLMI between children over time. Akaike's Information Criterion (AIC) was used to assess if an incremental model fitted the data better than the previous model (Burnham \& Anderson, 2002). Parental education level was not included as covariate in the series of models since a preliminary t-test showed no mean difference on RS between children of parents with and without a tertiary education degree $(\mathrm{t}=0.468$, $\mathrm{p}=0.640$ ); age was not included as covariate since the outcomes zFMI and zLMI are age-independent parameters, and moreover, inclusion of age did worsen the model fit. Hence, no covariates were included in the models. 
To rule out bias due to drop-out, sensitivity analyses were conducted by repeating the series of models on a subsample with zFMI and zLMI data at baseline and on the time point for which a significant cross-level interaction was present. The latter requirement was set and not the requirement of data on all time points in order to minimalize the reduction in sample size for the sensitivity analyses.

To illustrate the results graphically, the mean zFMI and zLMI of groups low and high in RS (lowest and highest tertiles respectively) were plotted as function of time.

\section{Results}

\section{Descriptive and drop-out statistics}

Table 1 shows descriptive statistics and parameter comparisons by sex. Only for zLMI, significant sex differences were present. Of the total study sample, $72.4 \%$ had minimum one tertiary educated parent, and $3.59 \%$ had missing data on parental education. At baseline, $8.1 \%$ of the children had overweight including obesity according to the International Obesity Task Force definition (Cole \& Lobstein, 2012).

Of the children with body composition data in 2011, (1) the baseline zFMI was significantly higher in those who dropped out versus those who participated in 2013 (mean difference 0.24 , 95\% confidence interval (CI) $[0.07,0.42], \mathrm{p}<0.01)$, and (2) the baseline age was significantly higher in those who dropped out versus those who participated in 2013 (mean difference 0.48 years, 95\% CI [0.21,0.75], p<0.01) and in 2015 (mean difference 0.54 years, 95\% CI [0.26,0.81], p<0.01). No other significant drop-out differences on RS, zFMI, zLMI, age, and sex were found. Time-constraints and decreased motivation of older children to participate in the study were the main responses of participants when the unwillingness to participate was questioned in telephone calls.

Explanation of between-child variability in the slopes of zFMI and zLMI over time by RS

Girls. Based on the AIC, model 4 of both zFMI and zLMI had the best data fit (Table 2). The results of model 4 demonstrated that RS significantly explained differences between girls (1) in baseline zFMI and zLMI, which is indicated by the significant positive coefficient of RS: with every one unit increase in RS, the baseline zFMI and zLMI of girls were 0.06 and 0.05 higher respectively; and (2) in the time slopes from baseline to 2015 
of zFMI and zLMI, which is indicated by the significant interaction between time variable 2015 (reference time 2011) and RS: with every one unit increase in RS, the zFMI and zLMI change from 2011 to 2015 of girls were 0.06 higher and 0.07 lower respectively. RS explained $14.8 \%$ and $11.6 \%$ of the variance in the zFMI and zLMI time slopes from baseline to 2015 (proportions are computed by subtracting the not rounded 2015-time slope variance of model 4 from that of model 3, and then dividing by the variance of model 3). Figure 2 displays the direction of these significant effects.

Sensitivity analyses on a subsample with body composition data on 2011 as well as 2015 (n=92, n measurements $=335)$ demonstrated a similar RS effect on the time slope from baseline to 2015 of $z F M I(p=0.03)$ and of zLMI, but the effect on zLMI was trend significant $(\mathrm{p}=0.09)$.

Besides these main findings, the intraclass correlation demonstrated that zFMI and zLMI were stable characteristics over time: $84.8 \%$ and $76.3 \%$ of the differences on zFMI and zLMI respectively were due to individual differences between girls, and consequently, only $15.2 \%$ and $23.7 \%$ of the differences on zFMI and zLMI respectively were due to differences between the measurement occasions (Table 2, table note). Additionally, variances in the zFMI and zLMI time slopes were only significant when the time span between the measurements was the largest, i.e. from 2011 to 2015 (model 3).

Boys. Based on the AIC, model 3 had the best data fit (Table 3). RS did not significantly explain baseline zFMI or zLMI nor differences in the time slopes of zFMI and zLMI between boys (model 4).

Besides these main findings, the intraclass correlation demonstrated that zFMI and zLMI were stable characteristics over time: $71.7 \%$ and $78.2 \%$ of the differences on zFMI and zLMI respectively were due to individual differences between boys, and consequently, $28.3 \%$ and $21.8 \%$ of the differences on zFMI and zLMI respectively were due to differences between the measurement occasions (Table 3, table note). Additionally, significant time slope variances on zFMI and zLMI were also only present between 2011 and 2015 (model 3).

\section{Discussion}

The current study is to our knowledge the first to examine the longitudinal association of scores on a general RS-questionnaire with fat and lean tissue accretion in boys and girls. RS is a personality trait, conceptualized here as a trait explaining differences in motivation to approach rewards in response to rewardpredicting-cues (Carver \& White, 1994). 
Girls but not boys with higher baseline RS had a significantly higher baseline relative fat mass and also experienced significantly more relative fat mass gain over time. These results suggest that natural variation in the trait RS contributes to the difference between remaining or not remaining on the same FMI percentile curve during growth in girls. However, the estimated increase in zFMI per unit increase in RS (i.e. 0.06), which equals an increase of 2.54 percentiles for children with a baseline zFMI of zero, appears to be relatively minor. The small change is possibly due to the use of a predominantly healthy-weight sample, resulting in less variety in FMI. Nevertheless, the relation between RS and zFMI change was significant, even in this sample. Further, the aim of the study was to identify appropriate targets for primary prevention of higher adiposity gain than expected on the basis of a child's age and height, and for this purpose, children that do not have a severe weight problem yet are suitable targets. Furthermore, RS can differ within a population over 12 units, and hence, the change in zFMI over time is estimated to differ with 0.72 points between girls at the lowest and highest extreme of RS. Moreover, RS explained a considerable amount of the variance in the zFMI change over four years, namely $14.8 \%$

Hence, the current results suggest that RS is a risk factor of higher fat tissue accretion than expected on the basis of their age and height. Thereby, RS was measured with a questionnaire that is easily and practically applicable for prevention purposes. The suggested increased risk for high RS girls is assumed to be explained by a higher vulnerability to palatable food cues in the environment (Paquet et al., 2010), followed by a higher motivation to obtain the food and resulting in higher consumption of these energy-dense foods (De Cock et al., 2016; De Decker et al., 2016). However, this was not tested in the current study.

The current results on the longitudinal association between a general RS-questionnaire and body composition cannot be compared to other studies in children since as far as we know, this longitudinal association has not been investigated yet. Cross-sectional studies on this association in children reported a positive association with BMI but did not consider sex differences (De Decker et al., 2016; Verbeken et al., 2012). In young adults, a longitudinal study did not find a significant relation between scores on a different general RS-questionnaire and FMI increase, but no sex differences were tested (Finlayson, Cecil, Higgs, Hill, \& Hetherington, 2012). One cross-sectional study in adults did consider sex differences and reported a similar finding, namely a positive association between RS and BMI in females but not males (Dietrich et al., 2009). As mentioned in the introduction, researchers have attempted to understand or measure cue-triggered reward motivation with various methods, other than general RS questionnaires. The to our knowledge available longitudinal studies with one of these methods in children are described below, but comparison with the current 
results has limitations since the methods differ drastically and do not necessarily measure the same construct. First, scores on a questionnaire that assesses to what extent a child is prepared to work towards obtaining food were found to predict increases in FMI (Hill, Saxton, Webber, Blundell, \& Wardle, 2009). In contrast with the questionnaire used in the current study, the child questionnaire used in the study in children is less applicable for prevention purposes since individual guidance is needed (Hill et al., 2009) and the validity is lower in younger children (French, Epstein, Jeffery, Blundell, \& Wardle, 2012). Second, no significant relation was found between reward circuitry responsivity to palatable food cues and onset of overweight or obesity (Stice, Yokum, \& Burger, 2013). However, both studies did not differentiate by sex. In sum, comparison of the current results with previous studies is difficult due to the use of different measurement approaches and/or populations as well as the lack of investigating sex-differences.

The most striking finding of the present study was that girls but not boys higher in RS exhibited higher relative lean mass at baseline, but longitudinal higher relative loss of lean mass. This suggests that natural variation in the trait RS also contributes to the difference between remaining or not remaining on the same LMI percentile curve during growth in girls. Thereby, a 0.07 lower zLMI change over four years was seen with every one unit increase in RS, which is equal to 1.99 percentiles for children with a baseline zLMI of zero. Although this is rather minor, RS explained $11.6 \%$ of the variance in zLMI change, which indicates that even in a predominantly healthy-weight sample, this trait may be relevant for body composition changes during growth.

There is a paucity of studies on this association. In a mixed sample of young adults, longitudinal changes in LMI were not significantly associated with scores on a general RS questionnaire, but positively associated with scores on a food-specific RS questionnaire (Finlayson et al., 2012). However, methodological differences obstruct us from comparing these findings with the current results.

Generally, fat accretion is accompanied by lean mass accretion, since more muscle mass is needed to carry a heavier body (Wells et al., 2006). However, in the current study, the opposite is seen. Since this outcome has not been reported previously, explanations of this finding remain speculative. Possibilities are that high RS girls are hampered in their activity level by the increasing adiposity or have a stronger decrease in physical activity with age. Related to the latter is the trend of a much steeper decrease in physical activity with every year of age in girls compared to boys (Corder et al., 2016). However, these speculations need to be confirmed in future research, since this was not tested in the current study.

Underlying reasons for the sex-specific RS effect on zFMI and zLMI are not completely clear since these were not investigated and sex differences in food reward and eating behavior still represent a developing 
line of research (Figlewicz, 2015). Literature suggests that biological factors may play a role. For example, patterns of neural activation to palatable food-cues differ by sex (Asarian \& Geary, 2013; Atalayer et al., 2014; Uher, Treasure, Heining, Brammer, \& Campbell, 2006). Another example is that the distribution of fat and lean mass changes differentially in boys and girls during growth; e.g. in boys but not girls, lean mass accretion is the primary target of weight gain during puberty since lean mass is a secondary sexual characteristic (Wells et al., 2012; Wisniewski \& Chernausek, 2009). Further, social factors may play a role. An example is the higher internalization of societal pressure to modify physical appearance, the higher body dissatisfaction and the higher drive for thinness in females compared to males (Yean et al., 2013). These factors compose a risk to develop disordered eating behavior via increases in weight control intentions (Stice \& Shaw, 2002).

Limitations of the study include the lack of information on puberty status, since this hampers the interpretation of the changes in body composition: although the zFMI and zLMI parameters capture the average sex-specific and percentile-specific alterations in the balance between fat and lean mass with age, children may enter puberty earlier or later than average. Another limitation is the use of British FMI and LMI reference data due to absence of Flemish reference data. Nonetheless, only minor differences between neighboring British and Flemish populations were seen (standard deviation of zFMI and zLMI on all time points close to one in both sexes). Comparable with the average zBMI based on Flemish reference scores, the average zFMI and zLMI were slightly negative, probably due to an overrepresentation of children from highly educated parents and therefore, healthier body composition. This is a limitation since it reduces the variation in zFMI and zLMI. Further, the relatively high accretion of fat and low accretion of lean mass in high RS girls was not found over one or two years, likely because it is harder to detect proper body composition trajectories over short time periods. Additionally, a large amount of participants dropped-out during the study. Nevertheless, no drop-out differences were present on the main outcomes (no significant difference between 2011 and 2015 on zFMI and zLMI) and the sensitivity analyses confirmed the study findings. Next, the study did not consider food intake and physical activity, and therefore, the interpretation of the results remains speculative. Future research should include these factors and elucidate if the relation between RS and increased fat tissue accretion is mediated by food intake or physical activity behavior. Moreover, it is critical that future research includes data on puberty.

Strengths of this study include the use of FMI and LMI based on air-displacement plethysmography. This enabled us to capture the simultaneous zFMI increase and zLMI decrease in high RS girls. Due to these opposite patterns, RS did not explain the net change in zBMI. Hence, the zBMI measure is incapable to capture the less favorable body composition development seen in high RS girls, and therefore, it is not advised to use 
BMI in future research on this topic. Other strengths are the longitudinal design and modelling, the large community sample of children, the differentiation by sex, and the use of the short Drive-subscale to measure RS, which is applicable for prevention purposes. Additionally, scores on this subscale have been positively associated with neural responsiveness in the brain reward system to food reward cues in young adults (Beaver et al., 2006). In children, an association with responsiveness to food reward has to our knowledge not been investigated yet, but a positive association between Drive-scores and neural responsiveness to monetary reward has been reported in participants aged 8-27 years (Braams, van Duijvenvoorde, Peper, \& Crone, 2015).

The current study findings suggest that girls but not boys high in RS are more vulnerable to unfavorable body composition development, i.e. higher adiposity gain parallel with lower lean mass accretion than expected on the basis of their age and height. The improved understanding on the contribution of a psychobiological trait to the variability in adiposity gain in obesogenic environments may inform pediatricians, parents, and policy makers, and support initiatives to better contain palatable food cues in the daily living environment of children. Further, several scientists recommend a shift toward targeted preventive interventions that focus on particularly vulnerable populations (e.g. Silveira et al. (2016)). The current study points out that girls high in RS might be more vulnerable, and may encourage future research to elucidate which prevention approaches are most effective for girls high in RS to timely reverse the unfavorable body composition development. For example, future research may determine if strengthening inhibitory control skills is an effective approach for girls high in RS, since good inhibitory control capacities are suggested to counteract reward-driven behavior (Appelhans, 2009).

\section{Acknowledgments}

The authors want to thank the participating children and their parents for their voluntary participation. Further, we want to thank Inge Blancke for her voluntary help, and Mia Bellemans, employee of the Department of Public Health, Ghent University, for her help in the body composition data collection.

\section{Funding}

This work was supported by the research council of Ghent University, the Institute for the Promotion of Innovation through Science and Technology in Flanders (grant number SBO-120054), and the Research Foundation - Flanders (I. Sioen, grant number 1.2.683.14.N.00). These funders had no role in the design, data 
collection, analyses or writing of this article, or the decision to submit this article for publication. Further, this research received no grant from any commercial funding agency.

\section{References}

Appelhans, B. M. (2009). Neurobehavioral Inhibition of Reward-driven Feeding: Implications for Dieting and Obesity. Obesity, 17(4), 640-647. doi:10.1038/oby.2008.638

Asarian, L., \& Geary, N. (2013). Sex differences in the physiology of eating. American Journal of Physiology-Regulatory Integrative and Comparative Physiology, 305(11), R1215-R1267. doi:10.1152/ajpregu.00446.2012

Atalayer, D., Pantazatos, S. P., Gibson, C. D., McOuatt, H., Puma, L., Astbury, N. M., \& Geliebter, A. (2014). Sexually dimorphic functional connectivity in response to high vs. low energy-dense food cues in obese humans: An fMRI study. Neuroimage, 100, 405-413. doi:10.1016/j.neuroimage.2014.05.054

Beaver, J. D., Lawrence, A. D., Van Ditzhuijzen, J., Davis, M. H., Woods, A., \& Calder, A. J. (2006). Individual differences in reward drive predict neural responses to images of food. Journal of Neuroscience, 26(19), 5160-5166. doi:10.1523/jneurosci.0350-06.2006

Braams, B. R., van Duijvenvoorde, A. C. K., Peper, J. S., \& Crone, E. A. (2015). Longitudinal Changes in Adolescent Risk-Taking: A Comprehensive Study of Neural Responses to Rewards, Pubertal Development, and Risk-Taking Behavior. Journal of Neuroscience, 35(18), 7226-7238. doi:10.1523/jneurosci.4764-14.2015

Burnham, K. P., \& Anderson, D. R. (2002). Model selection and multimodel inference: A practical information-theoretic approach. (Second ed.). New York, NY: Springer.

Carnell, S., Benson, L., Pryor, K., \& Driggin, E. (2013). Appetitive traits from infancy to adolescence: Using behavioral and neural measures to investigate obesity risk. Physiology \& Behavior, 121, 79-88. doi:10.1016/j.physbeh.2013.02.015

Carver, C. S., \& White, T. L. (1994). Behavioral-Inhibition, Behavioral Activation, and Affective Responses to Impending Reward and Punishment - the Bis Bas Scales. Journal of Personality and Social Psychology, 67(2), 319-333.

Cole, T. J., \& Lobstein, T. (2012). Extended international (IOTF) body mass index cut-offs for thinness, overweight and obesity. Pediatric Obesity, 7(4), 284-294. doi:10.1111/j.20476310.2012.000064.x

Corder, K., Sharp, S. J., Atkin, A. J., Andersen, L. B., Cardon, G., Page, A., . . van Sluijs, E. M. (2016). Age-related patterns of vigorous-intensity physical activity in youth: The International Children's Accelerometry Database. Preventive medicine reports, 4, 17-22. doi:10.1016/j.pmedr.2016.05.006

Davis, C., \& Fox, J. (2008). Sensitivity to reward and body mass index (BMI): evidence for a non-linear relationship. Appetite, 50(1), 43-49.

Davis, C., Patte, K., Levitan, R., Reid, C., Tweed, S., \& Curtis, C. (2007). From motivation to behaviour: a model of reward sensitivity, overeating, and food preferences in the risk profile for obesity. Appetite, 48(1), 12-19.

Davis, C., Strachan, S., \& Berkson, M. (2004). Sensitivity to reward: implications for overeating and overweight. Appetite, 42(2), 131-138.

De Cock, N., Van Lippevelde, W., Vervoort, L., Vangeel, J., Maes, L., Eggermont, S., . . Van Camp, J. (2016). Sensitivity to reward is associated with snack and sugar-sweetened beverage consumption in adolescents. European journal of nutrition, 55(4), 1623-1632. doi:10.1007/s00394-015-0981-3 
De Decker, A., Sioen, I., Verbeken, S., Braet, C., Michels, N., \& De Henauw, S. (2016). Associations of reward sensitivity with food consumption, activity pattern, and BMI in children. Appetite, 100, 189-196. doi:10.1016/j.appet.2016.02.028

Dietrich, A., Federbusch, M., Grellmann, C., Villringer, A., \& Horstmann, A. (2014). Body weight status, eating behavior, sensitivity to reward/punishment, and gender: relationships and interdependencies. Frontiers in Psychology, 5. doi:10.3389/fpsyg.2014.01073

Dietrich, A., Riese, H., van Roon, A. M., Minderaa, R. B., Oldehinkel, A. J., Neeleman, J., \& Rosmalen, J. G. (2009). Temperamental activation and inhibition associated with autonomic function in preadolescents. The TRAILS study. Biological Psychology, 81(1), 67-73.

Fields, D. A., Hull, H. R., Cheline, A. J., Yao, M., \& Higgins, P. B. (2004). Child-specific thoracic gas volume prediction equations for air-displacement plethysmography. Obesity Research, 12(11), 1797-1804.

Figlewicz, D. P. (2015). Modulation of Food Reward by Endocrine and Environmental Factors: Update and Perspective. Psychosomatic Medicine, 77(6), 664-670. doi:10.1097/psy.0000000000000146

Finlayson, G., Cecil, J., Higgs, S., Hill, A., \& Hetherington, M. (2012). Susceptibility to weight gain. Eating behaviour traits and physical activity as predictors of weight gain during the first year of university. Appetite, 58(3), 1091-1098. doi:10.1016/j.appet.2012.03.003

Franken, I. H., \& Muris, P. (2005). Individual differences in reward sensitivity are related to food craving and relative body weight in healthy women. Appetite., 45(2), 198-201.

French, S. A., Epstein, L. H., Jeffery, R. W., Blundell, J. E., \& Wardle, J. (2012). Eating behavior dimensions. Associations with energy intake and body weight. A review. Appetite, 59(2), 541549. doi:10.1016/j.appet.2012.07.001

Hill, C., Saxton, J., Webber, L., Blundell, J., \& Wardle, J. (2009). The relative reinforcing value of food predicts weight gain in a longitudinal study of 7-10-y-old children. American Journal of Clinical Nutrition, 90(2), 276-281. doi:10.3945/ajcn.2009.27479

Hox, J. (2010). Multilevel Analysis. Techniques and Applications. (Second ed.). New York, NY: Routledge.

Johnson, A. W. (2013). Eating beyond metabolic need: how environmental cues influence feeding behavior. Trends in Neurosciences, 36(2), 101-109. doi:10.1016/j.tins.2013.01.002

Lobstein, T., Baur, L., \& Uauy, R. (2004). Obesity in children and young people: a crisis in public health. Obesity Reviews, 5 Suppl 1, 4-104. doi:10.1111/j.1467-789X.2004.00133.x

McCrory, M. A., Gomez, T. D., Bernauer, E. M., \& Mole, P. A. (1995). Evaluation of a new air displacement plethysmograph for measuring human body composition. Medicine \& Science in Sports \& Exercise, 27(12), 1686-1691.

Michels, N., Vanaelst, B., Vyncke, K., Sioen, I., Huybrechts, I., De Vriendt, T., \& De Henauw, S. (2012). Children's Body composition and Stress - the ChiBS study: aims, design, methods, population and participation characteristics. Archives of Public Health, 70(1), 17. doi:10.1186/07787367-70-17

Muthén, L. K., \& Muthén, B. O. (1998-2012). Mplus User's Guide. Los Angeles, CA: Muthén and Muthén.

Paquet, C., Daniel, M., Knauper, B., Gauvin, L., Kestens, Y., \& Dube, L. (2010). Interactive effects of reward sensitivity and residential fast-food restaurant exposure on fast-food consumption. American Journal of Clinical Nutrition, 91(3), 771-776.

Roelants, M., Hauspie, R., \& Hoppenbrouwers, K. (2009). References for growth and pubertal development from birth to 21 years in Flanders, Belgium. Annals of Human Biology, 36(6), 680-694.

Silveira, P. P., Gaudreau, H., Atkinson, L., Fleming, A. S., Sokolowski, M. B., Steiner, M., ... Dube, L. (2016). Genetic Differential Susceptibility to Socioeconomic Status and Childhood Obesogenic Behavior Why Targeted Prevention May Be the Best Societal Investment. Jama Pediatrics, 170(4), 359-364. doi:10.1001/jamapediatrics.2015.4253 
Singh, A. S., Mulder, C., Twisk, J. W., van, M. W., \& Chinapaw, M. J. (2008). Tracking of childhood overweight into adulthood: a systematic review of the literature. Obesity Reviews, 9(5), 474488.

Stice, E., \& Shaw, H. E. (2002). Role of body dissatisfaction in the onset and maintenance of eating pathology - A synthesis of research findings. Journal of Psychosomatic Research, 53(5), 985993. doi:10.1016/s0022-3999(02)00488-9

Stice, E., Yokum, S., \& Burger, K. S. (2013). Elevated Reward Region Responsivity Predicts Future Substance Use Onset But Not Overweight/Obesity Onset. Biological Psychiatry, 73(9), 869876. doi:10.1016/j.biopsych.2012.11.019

Uher, R., Treasure, J., Heining, M., Brammer, M. J., \& Campbell, I. C. (2006). Cerebral processing of food-related stimuli: Effects of fasting and gender. Behavioural Brain Research, 169(1), 111119. doi:10.1016/j.bbr.2005.12.008

Verbeken, S., Braet, C., Lammertyn, J., Goossens, L., \& Moens, E. (2012). How is reward sensitivity related to bodyweight in children? Appetite, 58(2), 478-483. doi:10.1016/j.appet.2011.11.018

Vervoort, L., Vandeweghe, L., Vandewalle, J., Van Durme, K., Vandevivere, E., Wante, L., . . Braet, C. (2015). Measuring Punishment and Reward Sensitivity in children and adolescents with a parent-report version of the Bis/Bas-scales. Personality and Individual Differences, 87, 272277. doi:10.1016/j.paid.2015.08.024

Wells, J. C. K. (2000). A Hattori chart analysis of body mass index in infants and children. International Journal of Obesity, 24(3), 325-329. doi:10.1038/sj.ijo.0801132

Wells, J. C. K., Fewtrell, M. S., Williams, J. E., Haroun, D., Lawson, M. S., \& Cole, T. J. (2006). Body composition in normal weight, overweight and obese children: matched case-control analyses of total and regional tissue masses, and body composition trends in relation to relative weight. International Journal of Obesity, 30(10), 1506-1513. doi:10.1038/sj.ijo.0803402

Wells, J. C. K., Williams, J. E., Chomtho, S., Darch, T., Grijalva-Eternod, C., Kennedy, K., . . Fewtrell, M. S. (2010). Pediatric reference data for lean tissue properties: density and hydration from age 5 to 20 y. American Journal of Clinical Nutrition, 91(3), 610-618.

Wells, J. C. K., Williams, J. E., Chomtho, S., Darch, T., Grijalva-Eternod, C., Kennedy, K., . . Fewtrell, M. S. (2012). Body-composition reference data for simple and reference techniques and a 4component model: a new UK reference child. American Journal of Clinical Nutrition, 96(6), 1316-1326. doi:10.3945/ajcn.112.036970

Wisniewski, A. B., \& Chernausek, S. D. (2009). Gender in childhood obesity: family environment, hormones, and genes. Gender medicine, 6 Supp/ 1, 76-85. doi:10.1016/j.genm.2008.12.001

Yean, C., Benau, E. M., Dakanalis, A., Hormes, J. M., Perone, J., \& Timko, C. A. (2013). The relationship of sex and sexual orientation to self-esteem, body shape satisfaction, and eating disorder symptomatology. Frontiers in Psychology, 4, 887. doi:10.3389/fpsyg.2013.00887 


\section{Figure legends}

Figure 1. Number $(\mathrm{N})$ of participants with RS data at baseline and body composition data at baseline and followup.

Figure 2. The mean zFMI and zLMI of children low (lowest tertile, $\mathrm{n}$ girls=63, $\mathrm{n}$ boys=51) and high (highest tertile, $\mathrm{n}$ girls $=74, \mathrm{n}$ boys=84) in RS are displayed. In girls but not boys, baseline zFMI and zLMI significantly increased with RS, and the zFMI and zLMI change from 2011 to 2015 significantly differed by RS. The figure illustrates an increase in zFMI and a decrease in zLMI in the high RS group. zFMI; standard deviation score of Fat Mass Index based on British reference curves. zLMI; standard deviation score of Lean Mass Index based on British reference curves. RS; reward sensitivity. 
Table 1. Descriptive statistics of age, RS and body composition variables

\begin{tabular}{|c|c|c|c|c|c|c|c|c|}
\hline & \multirow[t]{2}{*}{ Year } & \multicolumn{3}{|c|}{ Boys } & \multicolumn{3}{|c|}{ Girls } & \multirow[t]{2}{*}{$P$ value $^{\mathrm{a}}$} \\
\hline & & $\mathrm{N}$ & Mean & sd & $\mathrm{N}$ & Mean & sd & \\
\hline Age (years) & 2011 & 223 & 8.83 & 1.51 & 223 & 8.87 & 1.45 & 0.79 \\
\hline RS (range 4-16) & 2011 & 223 & 8.90 & 2.90 & 223 & 8.44 & 2.80 & 0.09 \\
\hline \multirow[t]{4}{*}{ zBMI } & 2011 & 222 & -0.25 & 0.88 & 222 & -0.16 & 0.99 & 0.30 \\
\hline & 2012 & 158 & -0.34 & 0.89 & 160 & -0.18 & 0.92 & 0.12 \\
\hline & 2013 & 110 & -0.57 & 0.90 & 110 & -0.36 & 0.95 & 0.10 \\
\hline & 2015 & 81 & -0.46 & 0.87 & 92 & -0.21 & 0.91 & 0.07 \\
\hline \multirow[t]{4}{*}{ zFMI } & 2011 & 222 & -0.25 & 0.89 & 222 & -0.42 & 1.02 & 0.07 \\
\hline & 2012 & 158 & -0.33 & 0.94 & 160 & -0.41 & 0.92 & 0.47 \\
\hline & 2013 & 110 & -0.58 & 0.87 & 110 & -0.69 & 0.97 & 0.38 \\
\hline & 2015 & 81 & -0.28 & 0.95 & 92 & -0.49 & 0.97 & 0.15 \\
\hline \multirow[t]{4}{*}{ zLMI } & 2011 & 222 & -0.25 & 1.04 & 222 & -0.53 & 0.94 & $<0.01$ \\
\hline & 2012 & 158 & -0.31 & 0.98 & 160 & -0.49 & 0.90 & 0.08 \\
\hline & 2013 & 110 & -0.38 & 1.08 & 110 & -0.43 & 0.98 & 0.69 \\
\hline & 2015 & 81 & -0.70 & 1.08 & 92 & -0.38 & 1.00 & 0.05 \\
\hline
\end{tabular}

$\mathrm{Sd}$; standard deviation. RS; reward sensitivity. zBMI; standard deviation score of Body Mass Index based on Flemish reference curves. zFMI; standard deviation score of Fat Mass Index based on British reference curves. zLMI; standard deviation score of Lean Mass Index based on British reference curves.

${ }^{\mathrm{a}} \mathrm{P}$ value of unpaired t-test between boys and girls. 
Table 2. Fixed and random effects of two series of four incremental multilevel models with zFMI and zLMI as outcomes in girls $(n=223, n$ measurements on the four time points=584)

\begin{tabular}{|c|c|c|c|c|}
\hline & $\begin{array}{l}\text { Model } 1 \\
\mathrm{~b}(\mathrm{CI}) \\
\end{array}$ & $\begin{array}{l}\text { Model } 2 \\
\mathrm{~b}(\mathrm{CI}) \\
\end{array}$ & $\begin{array}{l}\text { Model } 3 \\
\mathrm{~b}(\mathrm{CI}) \\
\end{array}$ & $\begin{array}{l}\text { Model } 4 \\
\mathrm{~b}(\mathrm{CI}) \\
\end{array}$ \\
\hline \multicolumn{5}{|c|}{ Outcome: zFMI } \\
\hline \multicolumn{5}{|l|}{ Fixed effects } \\
\hline Intercept & $-0.41(-0.55,-0.28)^{* * *}$ & $-0.38(-0.51,-0.24)^{* * *}$ & $-0.38(-0.51,-0.25)^{* * *}$ & $-0.38(-0.52,-0.25)^{* * *}$ \\
\hline \multicolumn{5}{|l|}{ Time-level } \\
\hline \multicolumn{5}{|l|}{ Time $^{\mathrm{a}}$} \\
\hline 2012 & $-0.04(-0.10,0.03)$ & $-0.04(-0.11,0.03)$ & $-0.05(-0.11,0.02)$ & $-0.05(-0.12,0.02)$ \\
\hline 2013 & $-0.18(-0.27,-0.09)^{* * *}$ & $-0.18(-0.27,-0.09)^{* * *}$ & $-0.18(-0.28,-0.09)^{* * *}$ & $-0.18(-0.27,-0.08)^{* * * *}$ \\
\hline 2015 & $-0.09(-0.22,0.05)$ & $-0.09(-0.22,0.05)$ & $-0.09(-0.22,0.05)$ & $-0.06(-0.19,0.07)$ \\
\hline \multicolumn{5}{|l|}{ Child-level } \\
\hline $\mathrm{RS}^{\mathrm{b}}$ & & $0.07(0.02,0.11)^{* *}$ & $0.06(0.02,0.11)^{* *}$ & $0.06(0.01,0.11)^{*}$ \\
\hline \multicolumn{5}{|l|}{ Cross-level interaction } \\
\hline \multicolumn{5}{|l|}{$\operatorname{Time}^{\mathrm{a}} * \mathrm{RS}^{\mathrm{b}}$} \\
\hline 2012 & & & & $-0.01(-0.03,0.02)$ \\
\hline 2013 & & & & $0.01(-0.02,0.04)$ \\
\hline 2015 & & & & $0.06(0.01,0.11)^{*}$ \\
\hline \multicolumn{5}{|l|}{ Random effects } \\
\hline Time-level variance & $0.15(0.11,0.19)^{* * *}$ & $0.15(0.11,0.19)^{* * *}$ & $0.11(0.06,0.15)^{* * *}$ & $0.11(0.06,0.15)^{* * *}$ \\
\hline Child-level variance & $0.86(0.69,1.03)^{* * *}$ & $0.83(0.66,1.00)^{* * *}$ & $0.86(0.70,1.03)^{* * *}$ & $0.86(0.70,1.03)^{* * *}$ \\
\hline \multicolumn{5}{|l|}{ Time ${ }^{a}$ slope variance } \\
\hline 2012 & & & $<0.01(-0.08,0.09)$ & $<0.01(-0.08,0.09)$ \\
\hline 2013 & & & $0.02(-0.07,0.11)$ & $0.02(-0.07,0.11)$ \\
\hline 2015 & & & $0.22(0.05,0.39)^{*}$ & $0.19(0.03,0.34)^{*}$ \\
\hline AIC & 1162.70 & 1155.26 & 1134.50 & 1131.33 \\
\hline \multicolumn{5}{|c|}{ Outcome: zLMI } \\
\hline \multicolumn{5}{|l|}{ Fixed effects } \\
\hline Intercept & $-0.53(-0.65,-0.40)^{* * *}$ & $-0.50(-0.63,-0.38)^{* * *}$ & $-0.50(-0.62,-0.37)^{* * *}$ & $-0.50(-0.62,-0.37)^{* * *}$ \\
\hline \multicolumn{5}{|l|}{ Time-level } \\
\hline \multicolumn{5}{|l|}{ Time $^{a}$} \\
\hline 2012 & $0.04(-0.04,0.12)$ & $0.04(-0.04,0.12)$ & $0.03(-0.05,0.11)$ & $0.04(-0.04,0.11)$ \\
\hline 2013 & $0.09(-0.01,0.20)$ & $0.09(-0.01,0.20)$ & $0.09(-0.02,0.19)$ & $0.08(-0.02,0.19)$ \\
\hline 2015 & $0.18(0.02,0.34)^{*}$ & $0.18(0.01,0.34)^{*}$ & $0.18(0.02,0.34)^{*}$ & $0.15(-0.01,0.31)$ \\
\hline \multicolumn{5}{|l|}{ Child-level } \\
\hline $\mathrm{RS}^{\mathrm{b}}$ & & $0.045(<0.01,0.09)^{*}$ & $0.05(0.01,0.09)^{*}$ & $0.05(0.01,0.10)^{*}$ \\
\hline \multicolumn{5}{|l|}{ Cross-level interaction } \\
\hline \multicolumn{5}{|l|}{$\operatorname{Time}^{\mathrm{a}} * \mathrm{RS}^{\mathrm{b}}$} \\
\hline 2012 & & & & $0.01(-0.02,0.03)$ \\
\hline 2013 & & & & $-0.01(-0.04,0.03)$ \\
\hline 2015 & & & & $-0.07(-0.12,-0.01)^{*}$ \\
\hline \multicolumn{5}{|l|}{ Random effects } \\
\hline Time-level variance & $0.21(0.17,0.25)^{* * * *}$ & $0.21(0.17,0.25)^{* * *}$ & $0.14(0.08,0.21)^{* * *}$ & $0.14(0.08,0.21)^{* * *}$ \\
\hline Child-level variance & $0.68(0.54,0.83)^{* * *}$ & $0.67(0.53,0.81)^{* * *}$ & $0.69(0.55,0.82)^{* * *}$ & $0.68(0.55,0.82)^{* * *}$ \\
\hline \multicolumn{5}{|l|}{ Time $^{\mathrm{a}}$ slope variance } \\
\hline 2012 & & & $<0.01(-0.12,0.12)$ & $<0.01(-0.12,0.12)$ \\
\hline 2013 & & & $0.03(-0.06,0.12)$ & $0.03(-0.06,0.12)$ \\
\hline 2015 & & & $0.33(0.14,0.51)^{* * *}$ & $0.29(0.11,0.47)^{* *}$ \\
\hline AIC & 1239.08 & 1236.12 & 1208.69 & 1207.49 \\
\hline
\end{tabular}

b; unstandardized parameter estimate. CI; 95\% confidence interval. zFMI; standard deviation score of

Fat Mass Index based on British reference curves. zLMI; standard deviation score of Lean Mass Index based on British reference curves. RS; reward sensitivity, centered. AIC; Akaike’s Information Criterion.

Intraclass correlation (=not rounded child level variance/(child+time level variance)) based on model 1 for $\mathrm{zFMI}=84.8 \%$, for $\mathrm{zLMI}=76.3 \%$. 
${ }^{\mathrm{a}}$ Reference time of time dummies is $2011 .{ }^{\mathrm{b}}$ Range -5 to 7 . $* \mathrm{p}<0.05, * * \mathrm{p}<0.01, * * * \mathrm{p}<0.001$. 
Table 3. Fixed and random effects of two series of four incremental multilevel models with zFMI and zLMI as outcomes in boys $(n=223, n$ measurements on the four time points $=571)$

\begin{tabular}{|c|c|c|c|c|}
\hline & $\begin{array}{l}\text { Model } 1 \\
\mathrm{~b}(\mathrm{CI}) \\
\end{array}$ & $\begin{array}{l}\text { Model } 2 \\
\mathrm{~b}(\mathrm{CI}) \\
\end{array}$ & $\begin{array}{l}\text { Model } 3 \\
\mathrm{~b}(\mathrm{CI}) \\
\end{array}$ & $\begin{array}{l}\text { Model } 4 \\
\mathrm{~b}(\mathrm{CI})\end{array}$ \\
\hline \multicolumn{5}{|c|}{ Outcome: zFMI } \\
\hline \multicolumn{5}{|l|}{ Fixed effects } \\
\hline Intercept & $-0.26(-0.38,-0.14)^{* * *}$ & $-0.26(-0.38,-0.14)^{* * *}$ & $-0.26(-0.38,-0.14)^{* * *}$ & $-0.26(-0.38,-0.14)^{* * *}$ \\
\hline \multicolumn{5}{|c|}{ Lowest level: time-level } \\
\hline \multicolumn{5}{|l|}{ Time $^{\mathrm{a}}$} \\
\hline 2012 & $-0.03(-0.12,0.05)$ & $-0.03(-0.11,0.05)$ & $-0.02(-0.10,0.05)$ & $-0.02(-0.10,0.05)$ \\
\hline 2013 & $-0.19(-0.31,-0.07)^{* *}$ & $-0.19(-0.31,-0.07)^{* *}$ & $-0.17(-0.29,-0.06)^{* *}$ & $-0.17(-0.29,-0.05)^{* *}$ \\
\hline 2015 & $0.08(-0.10,0.26)$ & $0.08(-0.10,0.26)$ & $0.09(-0.10,0.27)$ & $0.09(-0.10,0.27)$ \\
\hline \multicolumn{5}{|c|}{ Highest level: child-level } \\
\hline $\mathrm{RS}^{\mathrm{b}}$ & & $0.01(-0.03,0.05)$ & $0.01(-0.03,0.05)$ & $0.01(-0.03,0.05)$ \\
\hline \multicolumn{5}{|l|}{ Cross-level interaction } \\
\hline \multicolumn{5}{|l|}{ Time $^{\mathrm{a} *} \mathrm{RS}^{\mathrm{b}}$} \\
\hline 2012 & & & & $0.01(-0.02,0.03)$ \\
\hline 2013 & & & & $<0.01(-0.04,0.04)$ \\
\hline 2015 & & & & $-0.02(-0.08,0.04)$ \\
\hline \multicolumn{5}{|l|}{ Random effects } \\
\hline Time-level variance & $0.24(0.18,0.31)^{* * *}$ & $0.24(0.18,0.31)^{* * *}$ & $0.13(0.06,0.19)$ & $0.13(0.06,0.19)$ \\
\hline Child-level variance & $0.63(0.48,0.77)^{* * *}$ & $0.63(0.48,0.77)^{* * * *}$ & $0.69(0.54,0.85)$ & $0.70(0.54,0.85)$ \\
\hline \multicolumn{5}{|l|}{ Time ${ }^{\mathrm{a}}$ slope variance } \\
\hline 2012 & & & $0.03(-0.07,0.12)$ & $0.03(-0.07,0.12)$ \\
\hline 2013 & & & $0.13(>-0.01,0.26)$ & $0.13(>-0.01,0.26)$ \\
\hline 2015 & & & $0.46(0.11,0.80)^{* *}$ & $0.45(0.09,0.81)^{*}$ \\
\hline AIC & 1258.05 & 1259.92 & 1227.73 & 1232.52 \\
\hline \multicolumn{5}{|c|}{ Outcome: zLMI } \\
\hline \multicolumn{5}{|l|}{ Fixed effects } \\
\hline Intercept & $-0.25(-0.38,-0.11)^{* * *}$ & $-0.25(-0.38,-0.11)^{* * *}$ & $-0.24(-0.38,-0.11)^{* * *}$ & $-0.24(-0.38,-0.11)^{* * *}$ \\
\hline \multicolumn{5}{|c|}{ Lowest level: time-level } \\
\hline \multicolumn{5}{|c|}{ Time $^{\mathrm{a}}$} \\
\hline 2012 & $-0.02(-0.10,0.07)$ & $-0.02(-0.10,0.07)$ & $-0.02(-0.10,0.06)$ & $-0.02(-0.10,0.06)$ \\
\hline 2013 & $-0.10(-0.21,0.02)$ & $-0.10(-0.21,0.02)$ & $-0.10(-0.21,0.02)$ & $-0.10(-0.21,0.02)$ \\
\hline 2015 & $-0.50(-0.66,-0.33)^{* * *}$ & $-0.50(-0.66,-0.33)^{* * *}$ & $-0.50(-0.66,-0.33)^{* * *}$ & $-0.50(-0.67,-0.34)^{* * *}$ \\
\hline \multicolumn{5}{|c|}{ Highest level: child-level } \\
\hline $\mathrm{RS}^{\mathrm{b}}$ & & $0.03(-0.02,0.07)$ & $0.03(-0.02,0.07)$ & $0.03(-0.02,0.08)$ \\
\hline \multicolumn{5}{|l|}{ Cross-level interaction } \\
\hline \multicolumn{5}{|l|}{ Time $^{\mathrm{a} *} \mathrm{RS}^{\mathrm{b}}$} \\
\hline 2012 & & & & $-0.02(-0.05,0.01)$ \\
\hline 2013 & & & & $<0.01(-0.04,0.04)$ \\
\hline 2015 & & & & $-0.01(-0.06,0.04)$ \\
\hline \multicolumn{5}{|l|}{ Random effects } \\
\hline Time-level variance & $0.22(0.18,0.25)^{* * * *}$ & $0.22(0.18,0.25)^{* * * *}$ & $0.16(0.10,0.21)^{* * *}$ & $0.15(0.10,0.20)^{* * *}$ \\
\hline Child-level variance & $0.88(0.70,1.05)^{* * *}$ & $0.87(0.70,1.04)^{* * *}$ & $0.89(0.72,1.06)^{* * *}$ & $0.89(0.72,1.06)^{* * *}$ \\
\hline \multicolumn{5}{|l|}{ Time $^{\mathrm{a}}$ slope variance } \\
\hline 2012 & & & $0.01(-0.09,0.10)$ & $0.01(-0.09,0.10)$ \\
\hline 2013 & & & $0.07(-0.03,0.17)$ & $0.07(-0.03,0.17)$ \\
\hline 2015 & & & $0.28(0.10,0.46)^{* *}$ & $0.28(0.10,0.47)^{* *}$ \\
\hline AIC & 1280.48 & 1280.97 & 1272.76 & 1272.76 \\
\hline
\end{tabular}

b; unstandardized parameter estimate. CI; 95\% confidence interval. zFMI; standard deviation score of

Fat Mass Index based on British reference curves. zLMI; standard deviation score of Lean Mass Index based on British reference curves. RS; reward sensitivity, centered. AIC; Akaike’s Information Criterion.

Intraclass correlation (=not rounded child level variance/(child+time level variance)) based on model 1 for $\mathrm{zFMI}=71.7 \%$, for $\mathrm{zLMI}=78.2 \%$. 
${ }^{\mathrm{a}}$ Reference time of time dummies is $2011 .{ }^{\mathrm{b}}$ Range -5 to 7 .

${ }^{*} \mathrm{p}<0.05, * * \mathrm{p}<0.01, * * * \mathrm{p}<0.001$ 